RESEARCH PAPER

\title{
Adverse effects of smoke exposure on the upper airway
}

J M Samet

Tobacco Control 2004;13(Suppl I):i57-i60. doi: 10.1136/tc.2003.005454

\begin{abstract}
Objective: This paper reviews secondhand smoke (SHS) exposure and diseases and symptoms of the upper airway, including the sinuses. Risks to flight attendants, who were occupationally exposed until smoking was banned on all flights, are emphasised.

Data sources: A systematic database search was conducted; the US Surgeon General's reports and other major reviews were evaluated. Literature summarised by National Research Council (NRC) reports on the airline cabin environment are included.

Study selection: A limited number of research publications on adults were identified; these are included. Many studies cited by the NRC were never published and information is taken directly from the reports. Data extraction: Data from observational studies of cabin crews and the general public were extracted from surveys; exposure monitoring of cabin crews is reported. Data from controlled exposure studies are included; most are challenge studies using volunteers screened for sensitivity to SHS.

Data synthesis: Evidence shows that active and passive smoking cause upper airway diseases, including sinonasal and laryngeal cancers in adult active smokers. Experimental studies indicate that brief exposures to SHS result in nasal mucosa inflammation. However, direct evidence on sinusitis is limited.

Conclusions: Evidence does not show a strong connection between active smoking and sinusitis, and active smokers have substantial exposures to SHS. However, extrapolation of these studies to cabin crews needs to be cautious, as other environmental conditions may increase risk for upper airway disease and symptoms. Surveys of cabin crews, while flawed, consistently indicate high rates of upper airway symptoms.
\end{abstract}

Correspondence to: Samet, Department of Epidemiology, Johns Hopkins Bloomberg Schoo of Public Health, $615 \mathrm{~N}$ Wolfe Street, Suite W6041, Baltimore, MD 21205, USA: jsamet@jhsph.edu chronic sinusitis. Flight attendants, working during the era when smoking was allowed on airplanes, may have been placed at particular risk for sinus disease, given the levels of SHS in smoking sections of airplanes and the dissemination of SHS throughout the cabin and the many hours that they were exposed while in flight. In addition, the flight attendants were exposed to the notably dry air of the aircraft cabins, which can adversely affect the mucosa which line the upper airway, and to the many other chemicals, including ozone, that can be present in the cabin..$^{4}$

An additional basis for concern can be found in the extensive literature on the adverse effects of SHS exposure on the respiratory health of children. In addition to causing increased risk for lower respiratory infections in infants and young children, SHS exposure also causes acute and chronic middle ear disease. ${ }^{6}$ The risks for acute otitis media are increased by as much as $50 \%$ in some studies, and for middle ear disease by about $50 \%$, as estimated in the meta-analysis by Strachan and Cook. ${ }^{7}$ These associations in children have been reviewed and found to be causal by the California Environmental Protection Agency, the World Health Organization, and the UK Scientific Committee on Tobacco. ${ }^{8-10}$ Only a few studies have addressed sinusitis in children, providing mixed findings. ${ }^{11}{ }^{12}$ In a study of 91 children, Nguyen et al ${ }^{11}$ found no association with passive smoke exposure. In a cohort study in Jordan, a remarkably strong association was observed between sinusitis and the presence of a smoker in the family, but other factors were not considered. ${ }^{12}$

This paper reviews the topic of SHS exposure and diseases and symptoms of the upper airway, including the sinuses.

Abbreviations: CO, carbon monoxide; MS, mainstream smoke; SHS, secondhand smoke; SS, sidestream smoke 
Emphasis is placed on the likely risks to flight attendants who were occupationally exposed until smoking was finally banned on all domestic and most international flights. ${ }^{5}$ This group of workers experienced other environmental conditions that may also contribute to sinusitis. The air within the cabin of an airplane is dry, typically at a relative humidity of $10-20 \%$ at cruise altitude. ${ }^{5}$ The air may also contain ozone, formaldehyde, and other aldehydes, and particles. ${ }^{45}$ Additionally, with each ascent the air in the middle ear and sinuses expands and then air re-enters these cavities on descent. Blockage to the movement of air by swelling of the tissue lining the ostia can result in pain and other symptoms. Consideration of the potential of SHS to cause sinusitis and ear disease in flight attendants needs to take into account the possibilities of enhanced susceptibility from these other factors.

In preparing this review, a systematic search of relevant databases was carried out, along with a review of the US Surgeon General's reports and other major reviews on smoking and health. Even though the adverse effects of SHS exposure have been a focus for extensive investigation in children, the literature search for adults identified only a limited number of research publications. Additional literature has been summarised by the 1986 and 2002 reports of National Research Council ${ }^{4}$ committees on the airliner cabin environment and in the proceedings of a 1999 meeting that was edited by Nagda. ${ }^{13}$ Many of the studies cited in these volumes were never reported in the peer reviewed literature.

\section{PATHOPHYSIOLOGY}

The nose is a complex structure that has the functions of warming and humidifying ambient air and also of filtering particulate and gaseous pollutants in the inhaled air. Its shape forces a change in the direction of airflow so that larger particles are removed by impaction and its substantial surfaces, which include the plate-like turbinates, serve as a "scrubber" for water soluble gases. Larger particles, greater than several micrometres in aerodynamic diameter, and very small particles, $<100 \mu \mathrm{m}$ in aerodynamic diameter, are filtered out with high efficiency, while particles in the intermediate size range tend to pass through and to reach the lower airways and lung. The particles of SHS, with mass median aerodynamic diameter around $0.40 \mu \mathrm{m}$, are in this size range and hence reach the lung, causing both malignant and non-malignant respiratory diseases. ${ }^{6}$

The sinuses are cavities in the facial bones that have uncertain physiological function, even though frequently a cause of symptoms and a site for infection. The sinuses include the maxillary, ethmoid, frontal, and sphenoid sinuses. Both the nose and the sinuses are lined by a pseudocolumnar epithelium; the cells include mucus secreting goblet cells as well as ciliated columnar cells. The sweeping function of the cilia removes potentially injurious gases, adsorbed into the surface mucus and water and particles, including infectious organisms. The cells also include macrophages, lymphocytes, and plasma cells, also key to the defence of the respiratory tract through phagocytosis by macrophages and immunologic responses, both humoral and cellular. ${ }^{14}{ }^{15}$

The ears also have a complicated structure. The middle ear, which is best described as a gas pocket, ${ }^{16}$ connects to the upper airway, or nasopharynx, through the Eustachian tube, and gas passes periodically from the nasopharynx through the Eustachian tube to the middle ear. This gas is not atmospheric air, but shares the composition of expired air, as when a person swallows following the exhalation of oxygen depleted and carbon dioxide rich gas from the nasopharynx. To bring pressure in the middle ear to equal the atmospheric pressure, gas is transferred from the nasopharynx to the middle ear via the Eustachian tube. ${ }^{16}$ Interference with the functioning of the Eustachian tube through swelling and the resulting obstruction at the ostia of the tubes contributes to the development of otitis.

SHS might plausibly cause irritation of the upper respiratory tract and also increase risk for infection. As a critical locus for defence against inhaled agents, the nose and upper airway "scrub" soluble gases from the air passing through and filter particles in critical size windows as well. Some of the components of SHS are known to be irritants and to adversely affect the functioning of respiratory defence mechanisms; components of particular concern in this regard include the SHS particles in general as well as aldehydes, particularly formaldehyde, and acrolein. The latter is toxic to cilia, potentially reducing their effectiveness as a clearance mechanism. Although not studied specifically in relation to SHS and the upper airway, cigarette smoke exposure from active smoking has diverse effects on lung immunology. ${ }^{17} 18$ Whether SHS exposure affects local humoral and cellular immune responses is uncertain, however.

\section{OBSERVATIONAL STUDIES \\ Cabin crew}

A substantial literature documents the irritation and annoyance associated with exposure to SHS. The initial literature on this topic was reviewed in the 1986 US Surgeon General's report. ${ }^{19}$ The report reviewed surveys on symptoms in nonsmokers exposed to SHS. Based on these reports, and supporting experimental evidence, a conclusion was reached that "The main effects of the irritants present in ETS occur in the conjunctiva of the eyes and the mucous membranes of the nose, throat, and lower respiratory tract."

Literature directly on SHS exposure and sinonasal symptoms and diseases is quite limited, however. The two National Research Council reports ${ }^{45}$ on the airline cabinet environment and the symposium proceedings edited by Nagda ${ }^{13}$ list most of the evidence available. Nagda and Koont ${ }^{20}$ have summarised the studies of flight attendant health and comfort in airliner cabins, identifying 21 papers; they point out substantial flaws in this body of literature and offer suggestions for future research.

The 1986 National Research Council ${ }^{4}$ report summarised 297 complaints by passengers of flights attendants compiled by the Association of Flight Attendants; "smoky" was given as a complaint in 73 of the total of 297 complaints, but causes were given for only 113. The report also refers to a 1980 questionnaire study of 1961 Scandinavian Airlines System (SAS) flight attendants. The majority (69\%) reported being bothered by "smoky air" while only $4 \%$ said that they were not at all bothered by smoky air. The 2002 report gave a more extensive compilation of studies and found methodological limitations of many that were reported. Complaints related to the nose, sinuses, and throat were common in the survey participants who included cabin crew and passengers, but no specific associations with SHS exposure were reported and most of the studies were carried out after smoking had been either restricted or banned.

Several specific surveys were described in the proceedings of the 1999 symposium. ${ }^{13}$ In a 1988 survey of SAS flight attendants described by Space et al ${ }^{21}$ tobacco smoke was reported to cause "discomfort to a great extent" by $66 \%$ of cabin attendants. Upper airway complaints were also prominent but further analyses were not described. In a mid 1990s survey of Cathay Pacific cabin crews, upper airway complaints were also common but the investigators did not attempt to relate the symptoms to the cabin environment. ${ }^{22}$ Mattson et $\mathrm{l}^{23}$ studied symptoms and nicotine exposures of nine persons (five passengers and four flight attendants) on four commercial flights. The protocol included exposure 
monitoring for nicotine while in flight and a collection of urinary cotinine following the flight. Symptom responses were also tracked. Eye and nose symptoms were related to nicotine exposure and cotinine excretion. Sinus symptoms were not explicitly assessed and the sample size was small.

\section{General population}

Lieu and Feinstein ${ }^{24}$ used the Third National Health and Nutrition Examination Survey, carried out from 1988 to 1994, to assess active and passive smoking and self reported sinusitis or sinus problems. The analysis was limited by reliance on self report. Measures of passive smoking were not significantly associated with sinusitis while active smoking was associated with an increased risk of approximately $20 \%$.

\section{STUDIES OF CONTROLLED EXPOSURES TO SHS}

Investigators have long addressed the hypothesis that some persons may be susceptible to SHS, possibly through allergic mechanisms. Research on this hypothesis has assessed allergy to tobacco smoke components; challenge studies have also been carried out to determine if some persons with asthma or with upper airways symptoms have heightened sensitivity.

Studies involving challenge with SHS exposure are potentially limited by the impossibility of masking the agent to which participants are being exposed. For that reason, some studies have included objective markers of response or injury, along with reports of symptoms. For asthma, SHS exposure has been linked to exacerbation. ${ }^{8} 1025$ The hypothesis that a subgroup of persons with asthma is particularly sensitive has been addressed in several studies, in which persons with asthma and reported SHS sensitivity were challenged with SHS exposure in a chamber. ${ }^{26-28}$ These studies did not provide consistent evidence for the existence of heightened susceptibility to SHS, as identified by self report. $^{25}$

In a series of studies carried out a decade ago, Bascom and colleagues explored the response of the nose to inhalation of SHS. ${ }^{29-31}$ These studies are particularly relevant to sinus disease, as they explored physiological and biochemical responses to exposure to SHS. The participants in the studies were volunteers who were screened by questionnaire for reported sensitivity to SHS. In the 1991 report by Bascom et al, ${ }^{29}$ the volunteers were initially sought for an independent study and then asked questions on symptoms following ETS exposure. A combined index of ocular and nasal responses was created and two groups were selected for further studythe most $(n=10)$ and least symptomatic $(n=11)$. Atopic status was assessed by skin prick tests and participants were exposed to SHS generated by a smoking machine for 15 minutes at a target concentration of $45 \mathrm{ppm}$ carbon monoxide (CO). The participants were studied on two separate days, one with measurement of nasal resistance and spirometry and the other with nasal lavage to study mediator responses.

With regard to symptoms, the "SHS sensitive" group tended to have more symptoms of rhinorrhoea and nose and throat irritation than the "non-sensitive" group. Symptom responses to the two challenges were generally concordant. Nasal resistance also rose more in the SHS sensitive participants. Mediators of inflammation measured in the nasal lavage fluid were unchanged by the SHS exposure.

In a 1992 report, Willes et al ${ }^{31}$ used similar methods but recruited participants who experienced nasal symptoms on exposure to SHS. The 18 persons selected for the study gave a history of symptoms on exposure. As in the previous study, symptoms and nasal airways resistance were increased by exposure to SHS using a similar 15 minute exposure.
What this paper adds

This paper reviews the effects of secondhand smoke on the upper airways of flight attendants and others whose occupations involve spending many hours in an airline cabin.

Historical symptoms correlated moderately with those observed with the experimental exposure.

In a study reported in 1995, Bascom et al ${ }^{30}$ recruited 12 healthy non-smokers, half having SHS sensitivity by history. The degree of nasal responsiveness was assessed by an initial screening challenge with SHS at a $\mathrm{CO}$ concentration of $15 \mathrm{ppm}$ for one hour. Participants were classified by the degree of increase in nasal airways resistance with this challenge protocol. In the main protocol, nasal mucociliary clearance was measured by tracking the rate of clearance from the nose of a radioactively labelled colloid. The response of the clearance rate to SHS exposure was variable, with six participants having an increased clearance rate and three, all with a history of rhinitis on exposure to SHS, having decreased clearance.

In a further study, reported in $1998,{ }^{32}$ the same investigative group challenged 23 healthy non-smokers with SHS at a $\mathrm{CO}$ concentration of $15 \mathrm{ppm}$. There were significant increases in irritant and rhinitis symptoms with SHS exposure and nasal resistance also increased.

\section{SUMMARY AND SYNTHESIS}

The aircraft cabin environment has conditions that would be expected to contribute to symptoms and disease of the upper airway. The air is dry and contaminated by diverse pollutants, including SHS previously, and passengers and crew go through substantial changes in pressurisation of the cabin. Monitoring studies carried out in the past document, the substantial contributions of smoking to air pollution in the cabin and a number of surveys indicate that SHS caused discomfort in this location as it did in others. While the components of SHS can injure the respiratory epithelium, associations of SHS exposure on board airplanes with adverse outcomes involving the upper respiratory tract have been little studied. Fortunately, with few exceptions, SHS exposures no longer occur on airplanes, but as a consequence, judgments concerning the role of SHS in causing symptoms and diseases of the upper airway, including sinusitis, need to be based in indirect evidence, coming from knowledge of the pollution in the cabins, the toxicity of the SHS components, and the possibility of synergism between SHS and other environmental aspects of the cabin, and from other lines of epidemiological and clinical evidence.

The evidence clearly shows that active and passive smoking cause diseases of the upper airway, including sinonasal cancer and laryngeal cancer in adult active smokers, and ear disease in passively exposed children. The experimental studies of Bascom and colleagues indicate that brief exposures to SHS result in inflammation in the nasal mucosa and that some people may have heightened sensitivity.

Further research could be feasibly carried out. Exposure to SHS remains common and sinonasal disease and symptoms are frequent. Epidemiological studies, based on either case-control or cohort designs, could be readily carried out, and further human exposure studies are needed to follow up on the work of Bascom and colleagues. Unfortunately, in spite of the morbidity and diminished quality of life associated with sinusitis and rhinitis, relevant research has been scant, perhaps reflecting a lack of research funding. 


\section{REFERENCES}

1 American Academy of Allergy, Asthma and Immunology and Small, B. Sinusitis: more than just an infection. Asthma and Allergy Advocate 2003;5:28.

2 Ray NF, Baraniuk JN, Thamer M, et al. Healthcare expenditures for sinusitis in 1996: contributions of asthma, rhinitis, and other airway disorders. J Allergy Clin Immunol 1999:103:408-14.

3 US Department of Health and Human Services. The health consequences of involuntary smoking. A report of the Surgeon General, 1986. Rockville, Maryland: Public Health Service, Centers for Disease Control, 1986. (DHHS Publication No (CDC) 87-8398.)

4 National Research Council (NRC), Committee on Airliner Cabin Environment Safety Committee. The airliner cabin environment: air quality and safety. Washington DC: National Academy Press, 1986

5 National Research Council (NRC), Committee on Air Quality in Passenger Cabins of Commercial Aircraft, Board on Environmental Studies and Toxicology. The airliner cabin environment and the health of passengers and crew. Washington DC: National Academy Press, 2002.

6 Samet JM, Wang SS. Environmental tobacco smoke. In: Lippmann M, ed. Environmental toxicants: human exposures and their health effects. New York: Van Nostrand Reinhold Co, Inc, 2000:319-75

7 Strachan DP. Cook DG. Parental smoking, middle ear disease and adenotonsillectomy in children. Thorax 1998;53:50-6.

8 California Environmental Protection Agency (Cal EPA) and Office of Environmental Health Hazard Assessment. Health effects of exposure to environmental tobacco smoke. California Environmental Protection Agency, 1997.

9 World Health Organization. International consultation on environmental tobacco smoke (ETS) and child health. Consultation Report. Geneva: World Health Organization, 1999.

10 Scientific Committee on Tobacco and Health and HSMO. Report of the Scientific Committee on Tobacco and Health. London: The Stationary Office, 1998.

11 Nguyen KL, Corbett ML, Garcia DP, et al. Chronic sinusitis among pediatric patients with chronic respiratory complaints. J Allergy Clin Immunol 1993;92:824-30

12 Kakish KS, Mahafza T, Batieha A, et al. Clinical sinusitis in children attending primary care centers. Pediatr Infect Dis J 2000;19:1071-4.

13 Nagda NL. Air quality and comfort in airliner cabins. Chelsea: American Society for Testing and Materials, 2000.

14 Gershwin ME, Incaudo GA. Diseases of the sinuses. Totowa, New Jersey: Humana Press, 1996.

15 Donald PJ, Gluckman JL, Rice DH. The sinuses. New York: Raven Press, 1995.

16 Sade J, Ar A. The Eustachian tube. In: Ludman H, Wright T, eds. Diseases of the ear. London: Arnold Publishers, 1998:334-52.
17 US Department of Health and Human Services. The health benefits of smoking cessation. A report of the Surgeon General, 1990. Rockville, Maryland: Public Health Service, Centers for Disease Control, Office on Smoking and Health, 1990. (DHHS Publication No (CDC) 90-8416.)

18 Sopori ML, Kozak W. Immunomodulatory effects of cigarette smoke. J Neuroimmunol 1998;83:148-52.

19 US Department of Health and Human Services. The health consequences of smoking for women. A report of the Surgeon General, 1980. Rockville, Maryland: Office of the Assistant Secretary for Health, Office on Smoking and Health, 1980. (DHHS Publication No (CDC) 90-8416.)

20 Nagda NL, Koontz MD. Review of studies on flight attendant health and comfort in airliner cabins. Aviat Space Environ Med 2003;74:101-9.

21 Space DR, Johnson RA, Rankin WL, et al. The airplane cabin environment: past, present and future research. In: Nagda NL, ed. Air quality and comfort in airliner cabins. Chelsea: American Society for Testing and Materials, 2000:189-214.

22 Lee SC, Poon CS, Li XD, et al. Air quality measurements on sixteen commercial aircraft. In: Nagda NL, ed. Air quality and comfort in airliner cabins. Chelsea: American Society for Testing and Materials, 2000:45-58.

23 Mattson ME, Boyd G, Byor D, et al. Passive smoking on commercial airline flights. JAMA 1989;261:867-72.

24 Lieu JE, Feinstein AR. Confirmations and surprises in the association of tobacco use with sinusitis. Arch Otolaryngol Head Neck Surg 2000;126:940-6

25 Weiss ST, Utell MJ, Samet JM. Environmental tobacco smoke exposure and asthma in adults. Environ Health Perspect 1999:107(suppl 6):891-5.

26 Menon P, Rando RJ, Stankus RP, et al. Passive cigarette smoke-challenge studies: increase in bronchial hyperreactivity. J Allergy Clin Immunol 1992;89:560-6.

27 Menon PK, Stankus RP, Rando RJ, et al. Asthmatic responses to passive cigarette smoke: persistence of reactivity and effect of medications. J Allergy Clin Immunol 1991;88:861-9.

28 Stankus RP, Menan PK, Rando RJ, et al. Cigarette smoke-sensitive asthma: challenge studies. J Allergy Clin Immunol 1988;82:331-8.

29 Bascom R, Kulle T, Kagey-Sobotka A, et al. Upper respiratory tract environmental tobacco smoke sensitivity. Am Rev Respir Dis 1991;143:1304-11.

30 Bascom R, Kesavanathan J, Fitzgerald TK, et al. Sidestream tobacco smoke exposure acutely alters human nasal mucociliary clearance. Environ Health Perspect 1995; 103:1026-30.

31 Willes SR, Fitzgerald TK, Bascom R. Nasal inhalation challenge studies with sidestream tobacco smoke. Arch Environ Health 1992;47:223.

32 Willes SR, Fitzgerald TK, Permutt T, et al. Acute respiratory response to prolonged, moderate levels of sidestream tobacco smoke. J Toxicol Environ Health A 1998;53:193-209. 